\title{
Special issue on: Computational discrete optimization
}

\author{
Arie M. C. A. Koster ${ }^{1} \cdot$ Clemens Thielen $^{2}$ \\ Accepted: 16 September 2020 / Published online: 21 October 2020 \\ (c) The Author(s) 2020
}

\section{Mathematics Subject Classification 90-08 · 90C10 · 90C27}

In a world where decisions are made on the basis of ever-growing amounts of available data, computational efficiency is a key property of almost every modern optimization algorithm. As many optimization problems are NP-hard, computationally efficient algorithms cannot be expected in the general context. The development of algorithms that nevertheless perform good in practice (with respect to solution time and quality) is an important corner stone of the success of operations research methods in the last decades. These algorithms can be either problem-specific or general-purpose like branch and cut for (mixed) integer linear programming.

Accordingly, a high number of contributions at the 30th European Conference on Operational Research (EURO 2019) that took place in Dublin in June 2019 consider algorithm improvements and novel algorithms for the efficient (exact or heuristic) solution of optimization problems. This, in particular, holds for the streams on Mixed Integer Programming and Algorithms on Graphs. The aim of this special issue has been to highlight some of these high-quality contributions. Both theoretical and more applied manuscripts were welcome, provided the computational contribution was significant. Submissions were, however, not restricted to contributions from these streams or even from the conference.

In total, we received eight submissions for this special issue. Six of these submissions have been accepted to be published in this issue of EURO Journal on Computational Optimization. In the following, we briefly highlight the novelty of these articles:

Arie M. C. A. Koster

koster@math2.rwth-aachen.de

Clemens Thielen

clemens.thielen@tum.de

1 Lehrstuhl II für Mathematik, RWTH Aachen University, Pontdriesch 10-12, 52062 Aachen, Germany

2 TUM Campus Straubing for Biotechnology and Sustainability, Weihenstephan-Triesdorf University of Applied Sciences, Am Essigberg 3, 94315 Straubing, Germany 
- As many discrete optimization problems can be modeled as mixed integer linear programs (MILPs), improving the performance of MILP solvers instantly increases the solvability for a multitude of optimization problems. Besides branch and bound, cutting planes, and primal heuristics, presolve techniques have a significant impact on MILP solver performance. In Gemander et al. (2020), Gemander et al. consider a new hashing-based pairing mechanism to consider two rows or columns simultaneously. By this, formulations can be strengthened further and the solution times of larger instances can be decreased.

- Another aspect that negatively impacts the performance of MILP solvers is dual degeneracy, i.e., the presence of multiple optimal bases in linear programs. This issue has been rarely studied so far. Gamrath et al. (2020) study the presence of dual degeneracy in mixed integer linear programs by introducing a new measure. Computations show how dual degeneracy develops during the branch and bound process and the correlation between dual degeneracy and the number of branching candidates. The results have been included in the MILP solver SCIP.

- Cutting and sequence optimization problems arise in many industrial applications, but are often computationally hard to solve. Ackermann and Diessel (2020) consider an integrated packing and sequence-optimization problem arising in the production of glued laminated timber in sawmills, where wood beams of different height and length shall be packed into a sequence of pressing steps subject to certain packing constraints of the press and requirements on subsequent pressing steps. They develop a heuristic three-stage solution approach for this problem and demonstrate its computational efficiency in a comprehensive numerical study using realistic input data.

- Another problem that is directly motivated from a real-world application is considered by Recalde et al. (2020), who develop an exact approach for the multiconstraint graph partitioning problem. This problem arises as a subproblem in a practical application where stores within a city are to be partitioned into a fixed number of clusters in the context of consumer price monitoring for basic commodities in Ecuador. The authors develop two integer programming formulations as well as an exact algorithm based on branch and bound and cutting planes for the problem and show the efficiency of their approach in computational tests on real-world instances.

- On a more theoretical side, Althaus et al. (2020) generalize the Euclidean Steiner minimum tree problem by replacing the set of points to be connected by line segments. They show that an earlier two-phase approach for the classical version can be adapted to the new version. In this adaption, the second phase remains unchanged. Computational results show that the algorithm is more efficient than an approximation by sampling the segments.

- Finally, Medrano (2020) presents two fast algorithms for solving the complete vertex $p$-center problem with Euclidean real number distances, where the goal is to solve the $p$-center problem for all $p$ from 1 to $m$ for a given maximum number $m$ of facilities. This results in a trade-off curve between the number of facilities and the maximum distance from any demand to the closest facility. One of the presented algorithms builds upon a classical MIP formulation for the $p$ center problem by trimming variables, and the other one converts the problem to a 
location set covering problem. Both algorithms are shown to be computationally efficient on a variety of spatial data sets.

Funding Open Access funding enabled and organized by Projekt DEAL.

Open Access This article is licensed under a Creative Commons Attribution 4.0 International License, which permits use, sharing, adaptation, distribution and reproduction in any medium or format, as long as you give appropriate credit to the original author(s) and the source, provide a link to the Creative Commons licence, and indicate if changes were made. The images or other third party material in this article are included in the article's Creative Commons licence, unless indicated otherwise in a credit line to the material. If material is not included in the article's Creative Commons licence and your intended use is not permitted by statutory regulation or exceeds the permitted use, you will need to obtain permission directly from the copyright holder. To view a copy of this licence, visit http://creativecommons.org/licenses/by/4.0/.

\section{References}

Ackermann H, Diessel E (2020) A hierarchical approach for solving an integrated packing and sequenceoptimization problem in production of glued laminated timber. EURO J Comput Optim. https://doi. org/10.1007/s13675-020-00127-8

Althaus E, Rauterberg F, Ziegler S (2020) Computing Euclidean steiner trees over segments. EURO J Comput Optim. https://doi.org/10.1007/s13675-020-00125-w

Gamrath G, Berthold T, Salvagnin D (2020) An exploratory computational analysis of dual degeneracy in mixed-integer programming. EURO J Comput Optim. https://doi.org/10.1007/s13675-020-00130-z

Gemander P, Chen W-K, Weninger D, Gottwald L, Gleixner A, Martin A (2020) Two-row and two-column mixed-integer presolve using hashing-based pairing methods. EURO J Comput Optim. https://doi. org/10.1007/s13675-020-00129-6

Medrano FA (2020) The complete vertex $p$-center problem. EURO J Comput Optim. https://doi.org/10. 1007/s13675-020-00131-y

Recalde D, Torres R, Vaca P (2020) An exact approach for the multi-constraint graph partitioning problem. EURO J Comput Optim. https://doi.org/10.1007/s13675-020-00126-9

Publisher's Note Springer Nature remains neutral with regard to jurisdictional claims in published maps and institutional affiliations. 